\title{
Frozen vs. fresh cycles IVF outcomes: retrospective study from an Indonesian IVF centre
}

\author{
Wiryawan Permadi ${ }^{1,2}$, Hartanto Bayuaji ${ }^{1,2}$, Kevin Dominique Tjandraprawira ${ }^{*}$ (D), Dian Tjahyadi ${ }^{1,2}$, \\ Harris Harlianto ${ }^{2}$, Yanni Melliandari Achmad ${ }^{2}$, Nanang Winarto Astarto ${ }^{2}$ and Tono Djuwantono ${ }^{1,2}$
}

\begin{abstract}
Objective: To compare the live birth rates (LBR) and neonatal outcomes of frozen cycle in vitro fertilization (IVF) with fresh cycle IVF in the Indonesian population.

Results: This was retrospective study using secondary data of IVF patients at a private fertility centre. Study recruitment was between 3/8/2018 and 31/12/2019. Total sampling included all patients undergoing oocyte retrieval and embryo transfer within recruitment period. Patients undergoing fresh IVF cycles and frozen IVF cycles were compared. 351 patients were recruited: $68.1 \%$ (239/351) underwent fresh cycles and 31.9\% (112/351) frozen cycles. AMH was significantly higher in frozen cycle group $(p=0.04)$. Ovulatory disorder was significantly higher in frozen cycle group $(p=0.001)$. Among patients aged $\leq 30$, fresh cycle group had significantly higher $L B R(p=0.02)$. Among those with ovulatory disorder, LBR was significantly higher with frozen cycle. No significant LBR difference was noted with other infertility causes. When stratified according to pregnancy order, frozen cycle patients had significantly higher birth lengths $(p=0.03)$ but not length of gestation nor neonatal birthweights. There was no significant difference in the proportion of biochemical pregnancy resulting in LBR $(p=0.08)$. To conclude, frozen cycle provided higher LBR among patients with ovulatory disorder but fresh cycle was beneficial among patients aged $\leq 30$.
\end{abstract}

Keywords: Embryo transfer, Frozen embryo transfer, Fertilization in vitro, Live birth

\section{Introduction}

Assisted reproductive technology (ART) is available in Indonesia to help against infertility, including in vitro fertilization (IVF) and its associated more advanced techniques [1]. Regarding IVF, frozen embryo transfer (FET) has been deemed superior to fresh embryo transfer (ET) policy with regards to the primary outcomes, i.e. the live birth rate (LBR), and fewer complications, particularly ovarian hyperstimulation syndrome (OHSS) [2].

\footnotetext{
*Correspondence: kevin.tjandraprawira.18@alumni.ucl.ac.uk

${ }^{1}$ Department of Obstetrics and Gynaecology, Faculty of Medicine,

Universitas Padjadjaran, Bandung, Indonesia

Full list of author information is available at the end of the article
}

Frozen cycle has been advocated for distinct settings, such as for endometriosis-associated infertility, in which FET may improve implantation rates [3]. In another study, external maternal factors, such as body mass index (BMI), could modify IVF success among polycystic ovarian syndrome (PCOS) patients [4]. Currently, the debate persists and the lack of sufficiently robust evidence on LBR indicates that the superiority of the frozen strategy cannot be fully advocated yet.

There are so far limited data on the outcome of IVF cycles in Indonesia. Thus, this study aims to compare the LBR and the associated neonatal birthweights and birth lengths of frozen IVF cycles and fresh cycles. 


\section{Main text Methodology}

This was a retrospective study utilising secondary data from patient case notes as a clinical audit of IVF patients presenting to a private fertility center in Bandung, Indonesia. The study recruitment period ran between 3 August 2018 and 31 December 2019. We employed total sampling and included all patients undergoing oocyte retrieval and embryo transfer within the recruitment period. We excluded IVF patients having undergone oocyte retrieval before 3 August 2018 despite having their embryo transfer (ET) or frozen embryo transfer (FET) within the recruitment period.

Patient case notes and laboratory worksheets were retrieved to collect data on patient demographics and details on their procedures. Data on the embryo transfer were also collected, regarding cycle type, the number of embryos/blastocysts transferred and their grades. Embryo grading was done using the Gardner criteria. Patients were assigned to either cycle at the clinician's discretion. Fresh cycle was defined as oocyte retrieval followed by cleavage-stage or blastocyst transfer in the same cycle. Frozen cycle was defined as oocyte retrieval followed by cryopreservation of all embryos (cleavagestage embryos and/or blastocyst). Thawing was done in a subsequent cycle and the embryo(s) were transferred.

Beta-hCG levels were measured at 13/15 days postembryo transfer and a beta-hCG level of $55 \mathrm{ng} / \mathrm{mL}$ established biochemical pregnancy [5]. For those achieving biochemical pregnancy, they were followed up by phone by the clinical staff for their pregnancy. Delivery details and their neonatal data were recorded. Patient participation was censored on 31 December 2019.

The ethical clearance for this study was granted by the Health Research Ethics Committee of Universitas Padjadjaran with the following Approval Number 1131/ UN6.KEP/EC/2020. This study received no external funding.

\section{Statistical methods}

Chi-squared test or Fisher's exact test were performed on categorical variables, depending on their sample sizes. T-test or the Mann-Whitney U test was utilised when comparing numerical variables containing two groups of data. Significance was set at 0.05 .

Data was entered into a customized database on Microsoft ${ }^{\circledR}$ Excel for Mac v16.16.3 (Microsoft $^{\circledR}$, Redmond, USA). They were analysed using Statistical Product and Software Solutions (SPSS) for Mac v.23 (IBM Corp, Armonk, New York, USA).

\section{Results}

Between 3 August 2018 and 31 December 2019, there were 351 patients divided into 2 groups: 239 patients (68.1\%) undergoing fresh cycle IVF and 112 patients undergoing frozen cycle (31.9\%). Table 1 presents the demographics of our patients. There was not a significant difference in age among those undergoing fresh cycle vs. frozen cycle. Using Mann-Whitney U Test, patients undergoing fresh cycles had significantly lower $\mathrm{AMH}$ levels than those undergoing frozen cycles.

The frozen cycle group had significantly more patients with ovulatory disorder than the fresh cycle group (39.3\% (44/112) vs. $21.3 \%(51 / 239)$, p-value 0.001 ) but not tubal factor infertility, uterine factor infertility, endometriosis and male-factor infertility.

Whilst both groups did not differ significantly in the number of oocytes retrieved, the median number of mature oocytes of the frozen group was significantly higher (9 (5-16) then the fresh cycle group (8 (4-12), p-value 0.03 .

The frozen group had significantly more embryos $(4$ (3-7) vs. $3(2-5), p=0.005)$. Whilst the 2 groups had similar number of excellent embryos, the frozen group still had significantly more good embryos (2 (1-3) vs. 1 (0-2), p-value 0.006).

Of those patients whose embryos were cultured into blastocysts, the 2 groups didn't differ significantly in the number of blastocysts produced, except that the frozen group had fewer good embryos than the fresh cycle group $(\mathrm{p}=0.04)$.

There was not a significant difference in the biochemical pregnancy rate of both groups $(p=0.7)$ but the LBR was significantly higher in the fresh-cycle group than the frozen group $(\mathrm{p}=0.05)$.

In Table 2, we found that among patients aged 30 years and less, the fresh cycle group had significantly higher LBR than the frozen group $(p=0.02)$. We found a statistically significantly higher LBR among those with ovulatory disorder treated with frozen cycle $(\mathrm{p}=0.04)$.

We assessed the neonatal outcomes of both groups (Table 3). Among singleton pregnancies, the birth length was statistically significantly higher among frozen cycle pregnancies $(p=0.03)$ but not the length of gestation nor the birth weight $(\mathrm{p}=0.08 ; 0.06)$. Among multiple pregnancies, none of the neonatal outcomes were significantly different between the two groups.

We found no significant LBR difference, regardless of whether the biochemical pregnancy came from a fresh ET or FET $(p=0.09)$. Only in fresh ET would the presence of excellent embryos significantly increase $\operatorname{LBR}(p=0.02)$. The association was not seen for excellent embryos towards live birth rate in FET $(\mathrm{p}=0.4)$. We also 
Table 1 Patient Demographics

\begin{tabular}{|c|c|c|c|}
\hline Variables & Fresh cycle (239) & Frozen cycle (112) & $p$-value \\
\hline Age (median, interquartile range) ${ }^{a}$ & $33(29-37)$ & $33(30-37)$ & 0.8 \\
\hline \multicolumn{4}{|l|}{ Abdominal surgery history ${ }^{\mathrm{b}}$} \\
\hline Yes & $127(53.1 \%)$ & $52(46.4 \%)$ & \multirow[t]{2}{*}{0.3} \\
\hline No & $111(46.9 \%)$ & $60(53.6 \%)$ & \\
\hline AMH level (median, interquartile range) ${ }^{a}$ & $2.02(1.3-3.28)$ & $2.54(1.32-4.7)$ & 0.04 \\
\hline \multicolumn{4}{|l|}{ Types of infertility ${ }^{\mathrm{b}}$} \\
\hline Primary infertility & $179(74.9 \%)$ & $79(70.5 \%)$ & \multirow[t]{2}{*}{0.4} \\
\hline Secondary infertility & $60(25.1 \%)$ & $33(29.5 \%)$ & \\
\hline Ovulatory disorder ${ }^{b}$ & $51(21.3 \%)$ & $44(39.3 \%)$ & 0.001 \\
\hline Tubal factor infertility ${ }^{\mathrm{b}}$ & $97(40.6 \%)$ & $37(33.0 \%)$ & 0.2 \\
\hline Uterine factor infertility ${ }^{b}$ & $61(25.5 \%)$ & $38(33.9 \%)$ & 0.1 \\
\hline Endometriosis $^{b}$ & $37(15.5 \%)$ & $16(14.3 \%)$ & 0.8 \\
\hline Male-factor infertility ${ }^{\mathrm{b}}$ & $183(76.6 \%)$ & $87(77.7 \%)$ & 0.8 \\
\hline \multicolumn{4}{|l|}{ Protocol $^{b}$} \\
\hline Long-protocol & $163(68.2 \%)$ & $65(58 \%)$ & \multirow[t]{2}{*}{0.06} \\
\hline Short-protocol & $76(31.8 \%)$ & $47(42 \%)$ & \\
\hline Oocytes retrieved (median, range) ${ }^{a}$ & $9(5-14)$ & $10(6-17)$ & 0.05 \\
\hline Mature oocytes (median, range) ${ }^{\mathrm{a}}$ & $8(4-12)$ & $9(5-16)$ & 0.03 \\
\hline Embryos number (median, range) ${ }^{a}$ & $3(2-5)$ & $4(3-7)$ & 0.005 \\
\hline Excellent embryos count (median, range) $^{a}$ & $2(1-3.5)$ & $2(1-4)$ & 0.8 \\
\hline Good embryos count (median, range) ${ }^{a}$ & $1(0-2)$ & $2(1-3)$ & 0.006 \\
\hline Blastocysts number (median, range) ${ }^{a}$ & $3(3-5)$ & $4(3-6)$ & 0.1 \\
\hline Excellent blastocysts count (median, range) ${ }^{\mathrm{a}}$ & $2(1-3)$ & $2(1-4)$ & 0.8 \\
\hline 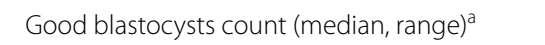 & $1(0-2)$ & $1(0-2)$ & 0.04 \\
\hline \multicolumn{4}{|l|}{ Biochemical pregnancy ${ }^{b}$} \\
\hline Yes & $71(29.7 \%)$ & $44(39.3 \%)$ & \multirow[t]{2}{*}{0.04} \\
\hline No & $168(70.3 \%)$ & $62(60.7 \%)$ & \\
\hline \multicolumn{4}{|l|}{ Live birth ${ }^{b}$} \\
\hline Yes & $57(23.9 \%)$ & $16(14.3 \%)$ & \\
\hline No & $182(76.1 \%)$ & $96(85.7 \%)$ & 0.05 \\
\hline
\end{tabular}

${ }^{a}$ Mann-Whitney $U$ test was used for continuous variables

${ }^{\mathrm{b}}$ Fisher's exact test was used for categorical variables

failed to observe the same association between excellent blastocysts and the LBRs $(\mathrm{p}=0.06)$.

\section{Discussion}

We found statistically significantly higher LBR among the fresh-cycle patients. Whilst our frozen cycle group is considerably smaller than the other group, our result agrees with Stormlund et al's study [6]. They found that among regularly menstruating women, frozen strategy did not improve the LBR when compared to fresh cycle strategy [6]. Their findings warrant caution towards the use of liberal frozen cycle strategy in the absence of strong indications such as ovarian hyperstimulation syndrome (OHSS) and preimplantation genetic testing (PGT) [6].
Our study found that among patients with ovulatory disorder, there was a significantly different proportion in LBR between the fresh cycle group $(10 / 47,17.5 \%)$ and the frozen group $(7 / 16,43.8 \%)(p=0.04)$. However, there was no significant difference in the biochemical pregnancy rate of the 2 groups $(\mathrm{p}=0.2)$. This is similar to Chen et al's study [7]. They found that among patients with polycystic ovarian syndrome (PCOS), FET resulted in significantly higher number of live births despite no significant difference in biochemical pregnancy rate [7]. This is a promising finding, as this would suggest that for those with PCOS, FET should be advocated to produce higher LBR. Furthermore, the relationship shown by Chen et al. was replicated in our study, despite our smaller number of subjects. 
Table 2 Live birth rate between groups

\begin{tabular}{|c|c|c|c|}
\hline \multirow[t]{2}{*}{ Variables } & \multicolumn{2}{|c|}{ Live birth rates among } & \multirow[t]{2}{*}{$\mathrm{p}=$ value } \\
\hline & Fresh cycle & Frozen cycle & \\
\hline Live birth rate $\leq 30$ year olds & $27(47.4 \%)$ & $2(12.5 \%)$ & \\
\hline Live birth rate $>30$ & $30(52.6 \%)$ & $14(87.5 \%)$ & 0.02 \\
\hline \multicolumn{4}{|l|}{ Ovulatory disorder ${ }^{\mathrm{a}}$} \\
\hline Present & $10(17.5 \%)$ & $7(43.8 \%)$ & \\
\hline Absent & $47(82.5 \%)$ & $9(56.2 \%)$ & 0.04 \\
\hline \multicolumn{4}{|l|}{ Tubal factor infertility ${ }^{\mathrm{a}}$} \\
\hline Present & $27(46.6 \%)$ & $4(25 \%)$ & 0.2 \\
\hline Absent & $30(53.4 \%)$ & $12(75 \%)$ & \\
\hline \multicolumn{4}{|l|}{ Uterine factor infertility ${ }^{a}$} \\
\hline Present & $14(24.6 \%)$ & $5(31.3 \%)$ & 0.8 \\
\hline Absent & $43(75.4 \%)$ & $11(68.7 \%)$ & \\
\hline \multicolumn{4}{|l|}{ Endometriosis $^{\mathrm{a}}$} \\
\hline Present & $9(15.8 \%)$ & $2(12.5 \%)$ & 1 \\
\hline Absent & $48(84.2 \%)$ & $14(87.5 \%)$ & \\
\hline \multicolumn{4}{|l|}{ Male-factor infertility ${ }^{\text {a }}$} \\
\hline Present & $47(82.5 \%)$ & $11(68.8 \%)$ & 0.3 \\
\hline Absent & $10(17.5 \%)$ & $5(31.2 \%)$ & \\
\hline
\end{tabular}

${ }^{a}$ Fisher's Exact test was used on the above categorical variables

We found that among patients with endometriosis, there was no significantly different proportion in the biochemical pregnancy and LBR between the groups ( $p>0.999 ; p=0.361$ respectively). Results have been conflicting. Mohamed et al. and Bourdon et al. reported that deferred embryo transfer through frozen strategy was significantly associated with higher cumulative pregnancy rate $[3,8]$. However, Feichtinger et al. reported the opposite [9]. Again, the lack of difference in our study might have been due to the lack of power as the number of endometriosis patients in our dataset was $<50$.

We discovered that there were roughly similar live birth rates among mothers $\leq 30$ years old and $>30$ years old in the fresh cycle group. However, in the frozen cycle group, mothers aged $>30$ years old significantly delivered more neonates than those $\leq 30$ years old. This is different from a very large-scale study in China by Zhu et al. [10]. Another study by Wang et al., though, could possibly corroborate our results [11]. They found that among women whose progesterone concentration $>1 \mathrm{ng} / \mathrm{mL}$, increasing age was associated with significantly higher LBR with freeze-only transfer cycles [11]. Whilst progesterone was not measured among our patients, the rising progesterone might have been the reason behind the very high success rates for frozen cycles among those $>30$ years old [11].

The lack of association between presence of excellent embryos and live birth rates in FET was surprising, despite the presence of such association among ET cycles. Whilst our lack of association is surprising, it might have been due to the relatively few pregnancies from FET patients over 1.5 years of data recruitment in our study. Another reason might have been due to suboptimal endometrium among our patients. With more patients opting for the frozen cycle at our facility, more data would be generated to allow for a repeat and more powerful analysis in the future.

The frozen cycle was associated with higher median birthweight (3200 (3009-3654) than the fresh cycle neonates (2800 (2200-3900)). Despite this association not being statistically significant, the trend was present and we suspect that the lack of significance was due to the fewer subjects of the frozen group when compared to the fresh cycle group. This was seen in the overall birthweight comparison, as the median birthweight of the freeze-cycle neonates (whilst not adjusting for the order of pregnancies) was statistically significantly higher than the fresh cycle neonates $(p=0.01)$. This may have been due to the good quality of the cryopreserved embryos [12]. Furthermore, the lower birthweight among fresh ETs might have been caused by the higher likelihood of abnormal placentation due to the over-estrogenized uterine environment [12].

Table 3 Neonatal outcomes among singleton and multiple pregnancies

\begin{tabular}{llll}
\hline Neonates from singleton pregnancies & Fresh cycle & Frozen cycle & p-value \\
\hline Length of gestation $^{\mathrm{a}}$ & $244.5(219-263)$ & $244(200-260)$ & 0.8 \\
Birth weight $^{\mathrm{a}}$ & $2800(2200-3900)$ & $3200(3009-3654)$ & 0.06 \\
Birth length $^{\mathrm{a}}$ & $48(44.5-52)$ & $49(45-51)$ & 0.03 \\
\hline Neonates from Multiple Pregnancies $^{\mathrm{N}}$ & Fresh cycle & Frozen cycle & $\mathbf{p}$-value \\
\hline Length of gestation $^{\mathrm{a}}$ & $231(224-235)$ & $228(222-232)$ & 0.6 \\
Birth weight $^{\mathrm{a}}$ & $2150(1835-2500)$ & $2545(222-2572.5)$ & 0.97 \\
Birth length $^{\mathrm{a}}$ & $45(44-47)$ & $47(45.5-47)$ & 0.97
\end{tabular}

${ }^{a}$ Mann-Whitney U Test was used for all above continuous variables 
Our study's strengths include the following. First, it is the first for our centre and to our knowledge, the first in our country to publish IVF data and compare the outcomes of fresh cycle IVFs against frozen-cycle IVFs. Second, our centre is among the top centres with high IVF cycles per year in Indonesia and our patients originate not just from the neighbouring cities but also from distant provinces.

To conclude, we did not find any significant differences in both the biochemical pregnancy rate and the LBR between frozen cycles and fresh cycles. We also failed to observe significant differences in the LBRs when stratified according to the aetiology of infertility except by their ages.

\section{Limitations}

However, our study has a number of limitations. Being the first in our country to compare the outcome of the 2 cycles, there are no data yet to compare our results with. Furthermore, our study is small, compared to the largescale studies and randomized controlled trials (RCTs) that have been published internationally and hence, the lack of power. Third, our study is single-centred thus the presence of selection bias and attrition bias cannot be ignored.

Furthermore, we didn't analyse the effect of the endometrial preparation on the success rate of FET pregnancies. With the limited dataset that we have, stratifying FET pregnancies according to their endometrial preparations would further reduce the power of the analysis. A bigger dataset would be necessary for a meaningful analysis.

We did not have data on potential confounding variables, such as patient BMI and smoking status and disabling adjustment for such confounding variables.

\section{Acknowledgements}

The authors would like to thank Miss Ike Kristina, Mr. Hafidz Shalahuddin and Miss Eva for their contribution in data collection.

\section{Authors' contributions}

WP, HBA and KDT conceived the study, $\mathrm{HH}$ and YMA collected the data. HBA and KDT performed the statistical analysis. DT, NWA and TD provided inputs and critique for the manuscript draft. All authors read and approved the final manuscript.

\section{Funding}

This study received no external funding.

\section{Availability of data and materials}

The datasets generated during this study are not available due to patient confidentiality reasons but are available upon reasonable written request to the corresponding author.

\section{Declarations}

\section{Ethical approval and consent to participate}

The ethical clearance for this study was granted by the Health Research Ethics Committee of Universitas Padjadjaran with the following Approval Number 1131/UN6.KEP/EC/2020.
Consent for publication

Not applicable.

\section{Competing interests}

The authors declare that we have no competing interests.

\section{Author details}

${ }^{1}$ Department of Obstetrics and Gynaecology, Faculty of Medicine, Universitas Padjadjaran, Bandung, Indonesia. Bandung Fertility Center, Limijati Women and Children Hospital, Bandung, Indonesia.

Received: 5 February 2021 Accepted: 23 April 2021

Published online: 10 May 2021

\section{References}

1. Bennett LR, Wiweko B, Hinting A, Adnyana IB, Pangestu M. Indonesian infertility patients' health seeking behaviour and patterns of access to biomedical infertility care: an interviewer administered survey conducted in three clinics. Reprod Health. 2012;9:24.

2. Blockeel C, Drakopoulos P, Santos-Ribeiro S, Polyzos NP, Tournaye H. A fresh look at the freeze-all protocol: a SWOT analysis. Hum Reprod. 2016;31(3):491-7.

3. Bourdon M, Santulli P, Maignien C, et al. The deferred embryo transfer strategy improves cumulative pregnancy rates in endometriosisrelated infertility: a retrospective matched cohort study. PLOS ONE. 2018;13(4):e0194800-e0194800.

4. Qiu M, Tao Y, Kuang Y, Wang Y. Effect of body mass index on pregnancy outcomes with the freeze-all strategy in women with polycystic ovarian syndrome. Fertil Steril. 2019;112(6):1172-9.

5. Bjercke S, Tanbo T, Dale PO, Mørkrid L, Åbyholm T. Human chorionic gonadotrophin concentrations in early pregnancy after in-vitro fertilization. Hum Reprod. 1999;14(6):1642-6.

6. Stormlund S, Sopa N, Zedeler A, et al. Freeze-all versus fresh blastocyst transfer strategy during in vitro fertilisation in women with regular menstrual cycles: multicentre randomised controlled trial. BMJ. 2020;370:m2519.

7. Chen Z-J, Shi Y, Sun Y, et al. Fresh versus frozen embryos for infertility in the polycystic ovary syndrome. N Engl J Med. 2016;375(6):523-33.

8. Mohamed AM, Chouliaras S, Jones CJ, Nardo LG. Live birth rate in fresh and frozen embryo transfer cycles in women with endometriosis. Eur J Obstet Gynecol Reprod Biol. 2011;156(2):177-80.

9. Feichtinger M, Nordenhök E, Olofsson Jl, Hadziosmanovic N, RodriguezWallberg KA. Endometriosis and cumulative live birth rate after fresh and frozen IVF cycles with single embryo transfer in young women: no impact beyond reduced ovarian sensitivity — a case control study. J Assist Reprod Genet. 2019;36(8):1649-56.

10. Zhu Q, Chen Q, Wang $L$, et al. Live birth rates in the first complete IVF cycle among 20687 women using a freeze-all strategy. Hum Reprod. 2018;33(5):924-9.

11. Wang A, Santistevan A, Hunter Cohn K, et al. Freeze-only versus fresh embryo transfer in a multicenter matched cohort study: contribution of progesterone and maternal age to success rates. Fertil Steril. 2017; 108(2):254-261.e254

12. Maheshwari A, Raja EA, Bhattacharya S. Obstetric and perinatal outcomes after either fresh or thawed frozen embryo transfer: an analysis of 112,432 singleton pregnancies recorded in the Human Fertilisation and Embryology Authority anonymized dataset. Fertil Steril. 2016;106(7):1703-8.

\section{Publisher's Note}

Springer Nature remains neutral with regard to jurisdictional claims in published maps and institutional affiliations. 